\title{
Comparison of F-Measure, BER and PSNR of Tumor Detection using Hybridization of Fuzzy and Region Growing
}

\author{
Simran Arora \\ M.Tech research scholar \\ Dept. of ECE, ACET Amritsar
}

\author{
Gurjit Singh \\ Assistant Professor \\ Dept. of ECE, ACET Amritsar
}

\author{
V.K. Banga, PhD \\ Principal \\ ACET Amritsar
}

\begin{abstract}
This paper has dedicated to brain tumor detection algorithm. The majority of the existing work with tumor detection has neglected the using object-based segmentation. Thus this paper has planned an effective brain tumor detection using the feature detection and roundness metric. To boost the tumor detection rate further we've incorporated the proposed hybridization of fuzzy C-means and region growing segmentation based tumor detection with the use of trilateral filter in its preprocessing stage. The planned method has the capability to generate efficient results even in the event of large occurrence of the noise. The experimental results have obviously shown that the planned method outperforms over the available techniques.
\end{abstract}

\section{Keywords}

Brain Tumor, Segmentation, Fuzzy C-means, Region growing, Trilateral filter.

\section{INTRODUCTION}

Image segmentation identifies the process of partitioning an image into mutually exclusive regions. It can be considered as the most essential and crucial process for facilitating the delineation, characterization, and visualization of regions of curiosity about any medical image. Despite intensive research, segmentation remains a complicated problem as a result of diverse image content, cluttered objects, occlusion, image noise, non-uniform object texture, and other factors. [1] Image segmentation was, is and is a major research topic for most image processing researchers. The reason why are obvious and applications endless: most computer vision and image analysis problems need a segmentation stage to be able to detect objects or divide the image into regions which is often considered homogeneous based on certain criterion, such as for instance color, motion, texture, etc[2]

\section{BRAIN TUMOR DETECTION}

A tumor might be main or secondary. If it is the source, then it is called primary. If the main tumor develops to another position and develops alone, then it is called secondary. Mental performance tumor influences CSF (Cerebral Spinal Fluid) and triggers strokes. The physician offers the therapy for the strokes as opposed to the therapy for tumors. So the detection of the tumor is important for that treatment. The life span of the person affected by mental performance tumor increases if it is detected at an earlier stage.[3]

A tumor is a mass of tissue that develops uncontrollable of the normal causes that regulates growth. The multifaceted mind tumors can be split in to two frequent groups relying on the tumors beginning, their enlargement prototype and malignancy. Primary mind tumors are tumors that get place commencing cells in the mind or commencing the wrapper of the brain. An inferior or metastatic mind tumor happens when cancer cells expand to the mind from a main cancer in a different element of the body. Many investigations in produced places show that the amount of people who develop mind tumors and depart that living from their store has higher than before maybe around 300 over previous three decades. The computationally efficient approach runs purchases of magnitude faster than current state of the artwork methods offering equivalent or improved results. Our quantitative effects suggest the advantage of incorporating design conscious affinities to the segmentation process for the hard case of mind tumor. That report conveys a well-organized method for computerized mind tumor segmentation for the removal of tumor areas from MR images. A effectively acknowledged segmentation trouble within MRI is the task of class voxels according for their tissue form which get bill of White Matter (WM), Gray Matter (GM), Cerebrospinal Fluid (CSF) and occasionally pathological areas like tumor etc. A mind tumor is an478 intracranial mass produced by an uncontrolled development of cells possibly commonly present in the mind such as neurons, lymphatic tissue, glial cells, body vessels, pituitary and pineal gland, brain, or distribute from cancers mostly located in other organs [4]. Brain tu mors are classified on the basis of the type of muscle involved, the located area of the tumor, whether it is benign or malignant, and different considerations. Primary (true) mind tumors are the tumors that begun in mental performance and are called for the mobile types from that they originated. They can be benign (non-cancerous), and thus they cannot escalation in a different place or strike neighboring tissues. They can be malignant and unpleasant (spreading to neighboring area). Extra or metastasis mind tumors take their source from tumor cells which raise to mental performance from a different position in the body. Most often cancers that raise to mental performance to purpose secondary mind tumors start in the lumy, breast, and kidney or from melanomas in the skin. [5].

On the other give, image segmentation algorithms are derived from among the two simple qualities of image intensity values: discontinuity and likeness [6]. In the conventional category, the segmentation approach is founded on dividing the processed image predicated on improvements in intensity, such as sides and corners. The next one is founded on dividing an image into regions that are related due to a group of predefined criteria. Thus, there are numerous segmentation techniques which may be extensively used, such as histogram based methods, edge-based methods, synthetic neural network based segmentation methods, bodily product based techniques, region-based methods (region breaking, growing, 
and merging), and clustering methods (Fuzzy C-means clustering, K-means clustering, Mean Shift, and Expectation Maximization) [7-9].

\section{LITERATURE SURVEY}

Abdel-Maksoud et al.[10] presented an efficient picture segmentation strategy applying K-means clustering process incorporated with Unclear C-means algorithm. It's been followed closely by thresholding and stage collection segmentation stages to supply exact brain tumor detection. The efficiency of the planned picture segmentation strategy was evaluated by comparing it with some state of the art segmentation algorithms in the case of accuracy, control time, and efficiency Havaei et al.[11] propose a semi-automatic strategy that sections a given brain by teaching and generalizing within that brain just, centered on some minimal user interaction. Investigate how e nearest neighbors $(\mathrm{kNN})$, likely the easiest device understanding strategy available, combined with easiest feature vector possible (raw MR indicate $+(\mathrm{x}, \mathrm{y}, \mathrm{z})$ position $)$ may be combined in to a technique that's simple, accurate and fast. Preetha et al.[12] presented that clustering of brain tumor images, applying Fuzzy D means is robust and efficient for tumor localization. Even although the proposed approach has high computational difficulty; it reveals remarkable results in segmentation effectiveness and convergence rate. The Fuzzy D means clustering with the expansion of Function removal and classification is extremely encouraging in the field of brain tumor detection. Dhanalakshmi et al.[13] uses pc served method for segmentation (detection) of brain tumor on the basis of the combination of two algorithms. This method allows the segmentation of tumor muscle with precision and reproducibility much like handbook segmentation. Additionally, in addition it decreases the full time for analysis. At the end of the procedure the tumor is removed from the MR picture and its precise position and the design also determined. The stage of the tumor is exhibited on the basis of the level of region determined from the cluster.Kumar et al. [14] examine about an improved segmentation algorithm for various defects detection in brain MRI (Tumor) and have compared the efficiency of this process with main-stream method. N. Albert Singh [15] presented an algorithm which features measures for preprocessing, function removal and classification using neural system techniques. The removal of structure characteristics in the noticed tumor has been achieved by using Gabor filter. These characteristics are used to teach and classify the mind tumor employing Artificial Neural System classifier. The system significantly improves the classification reliability of head tumor detection. $\mathbf{S}$. Parisot [16] propose a story method for detection, segmentation and characterization of head tumors. This process exploits previous knowledge in the shape of a short graph. Towards optimum usage of previous knowledge, a two layer interconnected chart is recognized as with one layer equivalent to the low-grade glioma type (characterization) and the second layer to voxel-based decisions of tumor presence. Effective linear programming both when it comes to performance as well as when it comes to computational load is recognized as to recover the cheapest possible of the objective function. The outcome of the strategy describes both tumor segmentation as well as their characterization. Bhattacharjee, Rupsa, et al.[17] In that paper, centered on a examine of quality parameter contrast of two filters, adaptive median filtration is selected for de-noising the images. Image slicing and recognition of significant airplanes are done. Rational procedures are used on selected cuts to obtain the prepared image featuring the tumor region. A novel image reconstruction algorithm is developed on the basis of the request of Key Parts Examination (PCA).Shasidhar et al. [18] propose the application of revised FCM algorithm for MR mind tumor detection. An extensive feature vector place is employed for the segmentation technique. Relative analysis in terms of segmentation efficiency and convergence charge is conducted between the conventional FCM and the revised FCM. Fresh benefits show superior benefits for the revised FCM algorithm in terms of the performance measures. Bhat et al. [19] propose the mix of watershed algorithm with productive contour algorithm to lessen the computational complexity and to improve the insensitiveness to noise. The real brain MR image is first segmented applying watershed segmentation and then edges made would be the initial contour of productive contour algorithm. The planned method is superior when it comes to record selection, concave tumor place extraction, segmentation precision and sensitivity to noise. Kannan et al. [20] gifts an altered FCM algorithm for segmentation of MRI. The proposed process has presented by adjusting the objective purpose of the standard FCM and it's the advantage that it could be applied at an earlier stage in a automated knowledge analysis. The proposed process can handle the strength in-homogeneities and image sound effectively.. Singh, Laxman et al. [21] present a method to characterize a brain tumor using Marker Controlled Watershed Segmentation method and region property functions using image processing toolbox. The parameters extracted are area, major and minor axis length, eccentricity, orientation, equivdiameter, solidity and perimeter. This method is quite versatile, fast and simple to use. This can be applied to all type of 2D MR Images representing all tumors irrespective of their location in human body and their size. Wu, Ming-Ni et al. propose a color-based segmentation approach that employs the K-means clustering approach to track tumor items in magnetic resonance (MR) brain images. The important thing principle in this color-based segmentation algorithm with $\mathrm{K}$-means is always to change certain graylevel MR picture in to a color place picture and then separate the positioning of tumor items from other components of an MR picture by using K- suggests clustering and histogramclustering. Experiments display that the technique can effectively achieve segmentation for MR brain images to greatly help pathologists distinguish precisely lesion measurement and region.

\section{THE PROPOSED MEDICAL IMAGE SEGMENTATION SYSTEM}

There are a few medical image segmentation techniques which use place rising process for sensing tumor in head [23]. The place rising method can effectively split up the regions that have exactly the same qualities and easy to run on large datasets however it suffers from issue of photographs with high occurrence of noise. On another hand, different techniques use Fuzzy C-means algorithm as it maintains the additional information of the initial image to discover malignant tumor cells precisely set alongside the K-means [24]. These techniques are painful and sensitive to noise and outliers and they take extended delivery time. Within our planned medical segmentation process, we get benefits from the final two algorithms. As found in Fig. 1, the planned medical image segmentation process includes hybridization of Fuzzy C means clustering with the region growing segmentation approach using trilateral filteration in its 


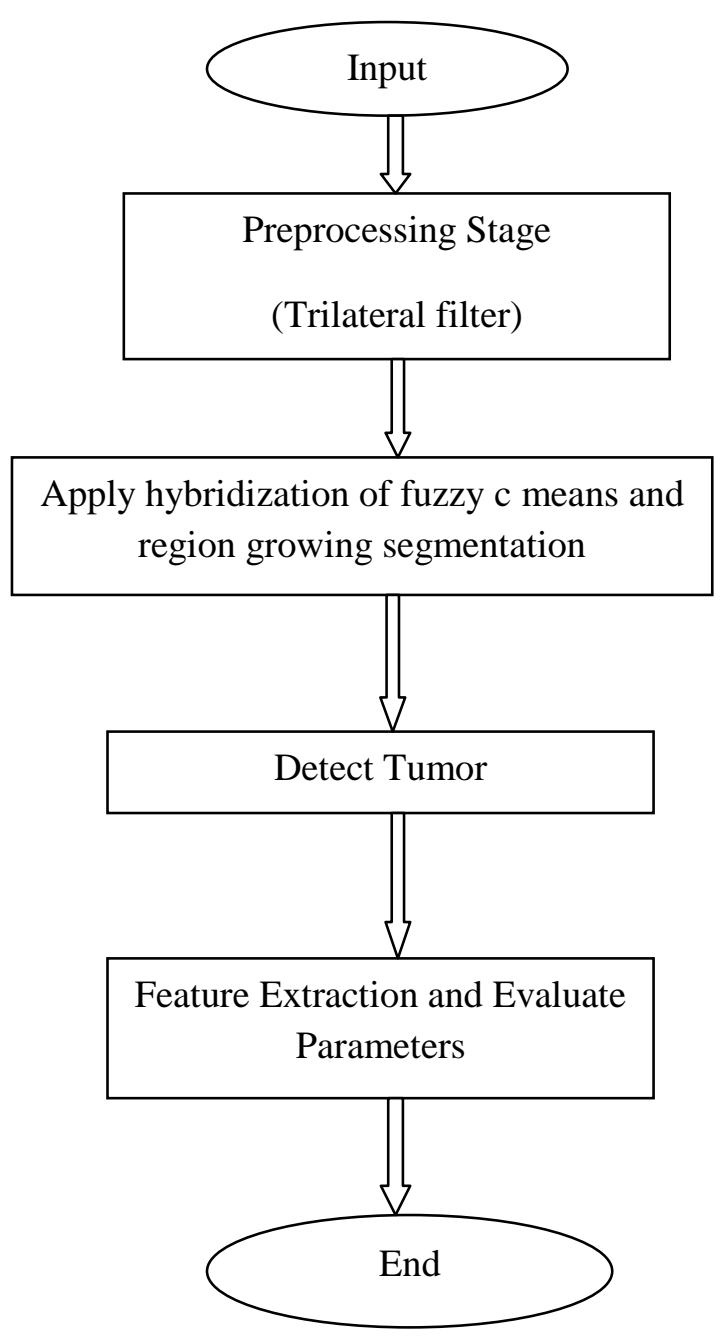

Fig. 1: Proposed Methodology

with the region growing segmentation approach using trilateral filtration in its Pre-processing stage. Moreover features are removed from the image using roundness matrix. The main strategy of doing the integration is to lessen the number of iterations done by initializing the proper bunch stores to Unclear C-means clustering methods that, obviously, diminishes delivery time and provide qualitative results. The results of our tests clarified our cross clustering approach may identify a tumor that cannot be noticed by Unclear C-means with less delivery time. The main phases of the proposed program are likely to be mentioned in greater detail in the following sections.

\subsection{Pre-processing stage}

According to the require of the following level the pre processing stage change the image. It performs selection of noise and different items in the image and maintenance the edges in the image. RGB to grey transformation and Reshaping also happens here.[13] This phase is applied by applying some original processing techniques on the image before any particular applications processing. It increases the image quality and eliminates the noise. Because, mental performance images tend to be more painful and sensitive than different medical images; they should be of minimum noise and optimum quality. Therefore, that stage contains the next filtration.

\subsubsection{Trilateral Filter}

It is really a nonlinear filtration proposed by Choudhry and Tumblin cope with HDR Tone Mapping, Comparison Reduction by mixing a gradient bilateral filtration and intensity bilateral filtration with a chart based method to restrict filtration window. Using clean gradient, they "tilt" the filtration screen of intensity bilateral filter. The expansion of intensity bilateral window restores the potency of spatial filtration term. All of the inner variables found in trilateral filtration produced from simple user-supplied value. Trilateral filtration applies a non-linear filtration to eradicate Gaussian noise while preserving the sharp, ridge-like and valley-like edges. By presenting the intuition alarm to the gradient bilateral filters, and pre-processing the feedback picture before intensity bilateral filtration to trilateral filtration, the trilateral filtration can very quickly eliminate the mix noise (Gaussian noise and impulsive noise). Compared to all or any or some other non-linear filters, trilateral filters gives better benefits in providing stronger noise reduction and side preservation. This kind of filters is found in MR images.

\subsection{Hybridization of Fuzzy C-means and Region growing segmentation}

By de-noising the MRI image and removing skulls, the images are fed to hybridization block. Clustering is one of many trusted image segmentation techniques which categorize designs in this way that types of the exact same class tend to be more related to one another than products owned by different teams [25]. There has been considerable interest recently in the utilization of fuzzy clustering strategies, which retain more info from the original image than difficult clustering methods. Unclear C-means algorithm is commonly chosen due to its additional flexibility which allows pixels to participate in numerous lessons with various examples of membership. Nevertheless the key working criticism is that the FCM technique is time consuming. The disadvantage of the FCM is increased by the increased FCM algorithm.

\subsubsection{Fuzzy C-means Clustering}

Fuzzy $\mathrm{C}$ means is a method of clustering which allows one pixel to belong to one or more clusters[26]. The FCM algorithm attempts to partition a finite collection of pixels into a collection of "C" fuzzy clusters with respect to some given criteria [27]. Fuzzy C-means Algorithm is based on minimization of the following objective function

$$
J\left(U, c_{1}, c_{2}, \ldots . c_{c}\right)=\sum_{i=1}^{c} J_{i}=\sum_{i=1}^{c} \sum_{j=1}^{n} u_{i j}^{m} d_{i j}^{2}
$$

$u_{i j}$ lies between 0 and $1 ; c_{i}$ is the centroid of cluster I; $d_{i j}$ is the Euclidean distance between $i^{\text {th }}$ centroids and $j^{\text {th }}$ data point; $\mathrm{m} \in[1, \infty]$ is a weighting function. Fuzzy portioning of the known sample of data is carried out through an iterative optimization of the objective function

$$
u_{i j}=\frac{1}{\sum_{k=1}^{c}\left(\frac{d_{i j}}{d_{k j}}\right)^{2 /(m-1)}} \quad c_{i j}=\frac{\sum_{j=1}^{n} u_{i j}^{m} x_{j}}{\sum_{j=1}^{n} u_{i j}^{m}}
$$

The iteration will stop when

$$
\max _{i j}\left\{\left|u_{i j}^{(k+1)}-u_{i j}^{(k)}\right|\right\}<\varepsilon \text {, }
$$


where $\varepsilon$ is a termination criterion between 0 and 1 , whereas $k$ are the iteration steps. The procedure converges to a saddle point or local minimum of $\mathrm{Jm}$.

The algorithm consists of the following steps:

1. Initialize $\mathrm{U}=\left[u_{i j}\right]$ matrix, $U^{(0)}$

2. At k-step: calculate the centers vectors $C^{(k)}=\left[c_{j}\right]$ with $U^{(k)}$

$C_{j}=\frac{\sum_{i=1}^{N} u_{i j}^{m} x_{i}}{\sum_{i=1}^{N} u_{i j}^{m}}$

1. Update $U^{(k)}, U^{(k+1)}$

$$
\left.U_{I J}=\frac{1}{\sum_{k=1}^{c}\left(\left\|x_{i}-c_{j}\right\|\right.}\right)^{2 / m-1}
$$

2. If $\left\|U^{(k+1)}-U^{(k)}\right\|<\varepsilon$ then STOP; otherwise return to step 2.[28]

\subsubsection{Region growing}

In region growing / region merging segmentation technique pixels with similar intensities are grouped. With a pixel or group of pixels referred to as seeds belonging to the framework in concentration, the first faltering step with this technique is started. Pixels in little community location are examined in the next phase and added to the growing location on the foundation of homogeneity criterion. Until you can forget pixels could be adjoined to the growing parts, this step continues. Eventually, the item illustration is completed by all included pixels to the growing regions.In the medical image segmentation subject location growing technique could be used in kidney segmentation, cardiac pictures, removal of brain area etc. The capacity of generating joined parts and appropriately segmenting parts having corresponding home are the advantages of that segmentation method. One of the disadvantages of this approach is that dissimilar starting factors might not outcome growing in to identical regions. As well as that, since result of location growing is determined by homogeneity criterion, failure in appropriately choosing criterion might result in adjoining places or parts perhaps not belonging to the item of interest. 'Hill climbing'which is really a adjustment of location growing segmentation strategy was requested distinguishing micro calcifications in mammograms. In slope climbing a mountain price $s(x, y)$ for each pixel is symbolized as

$$
s(x, y)=\frac{f\left(x_{0}, y_{\circ}\right)-f(x, y)}{d\left(x_{0}, y_{\circ}, x, y\right)}
$$

Here $f(x, y)$ refers to the prescribed image, pixel $\left(x_{\circ}, y_{0}\right)$ refers to the edge of a micro calcifications to be segmented and $\mathrm{d}\left(\mathrm{x}_{\circ}, \mathrm{y}_{\circ}, \mathrm{x}, \mathrm{y}\right)$ defines the Euclidean distance which exist between pixel $(x, y)$ and the local maximum pixel [29].

\subsection{Feature extraction and evaluate parameters}

Feature detection based tumor detection algorithm using roundness metric given in equation

$$
\begin{gathered}
\text { Metric }=4 * \prod * \text { area } / \text { perimeter }^{2} \\
\text { Perimeter }=\sqrt{\sum \mathrm{d}^{2}}
\end{gathered}
$$

Where $d$ is the estimate of the object's perimeter

\section{RESULTS}

INPUT IMAGE:

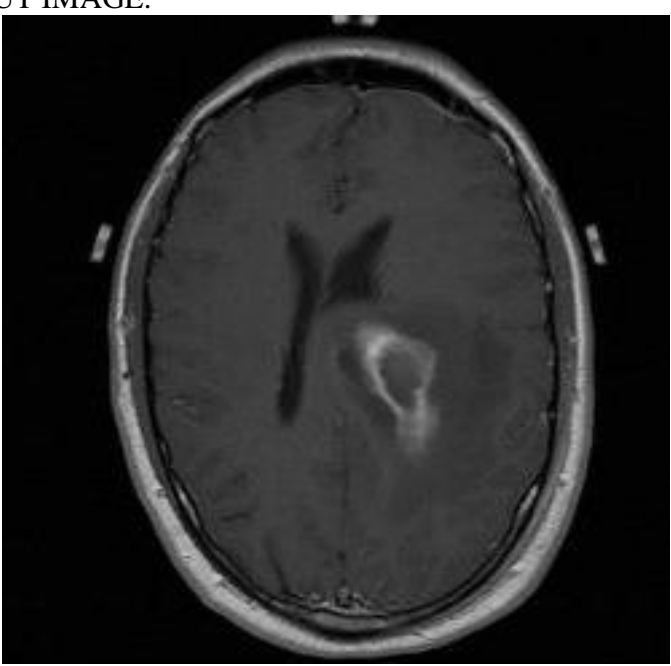

Fig 2: Input image

\section{BASE PAPER SEGMENTED IMAGE:}

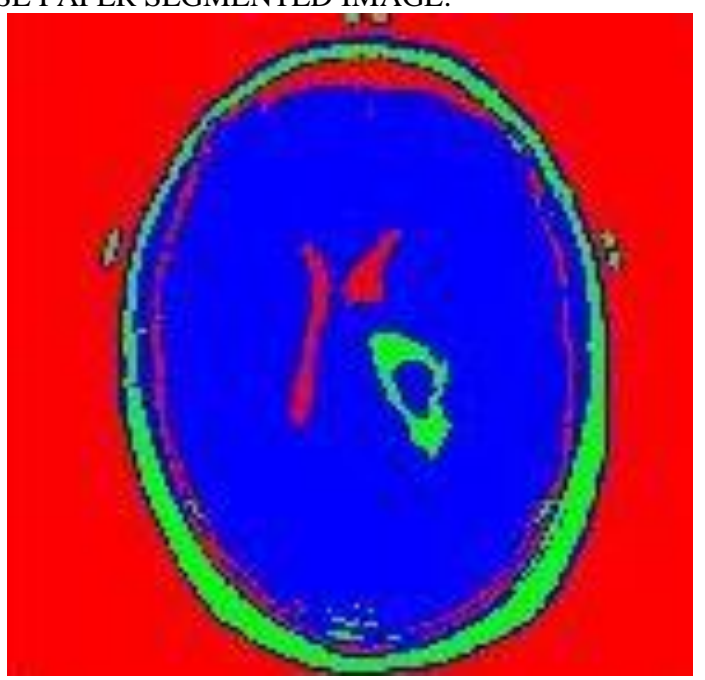

Fig 3:Base paper segmented image

PROPOSED SEGMENTED IMAGE:

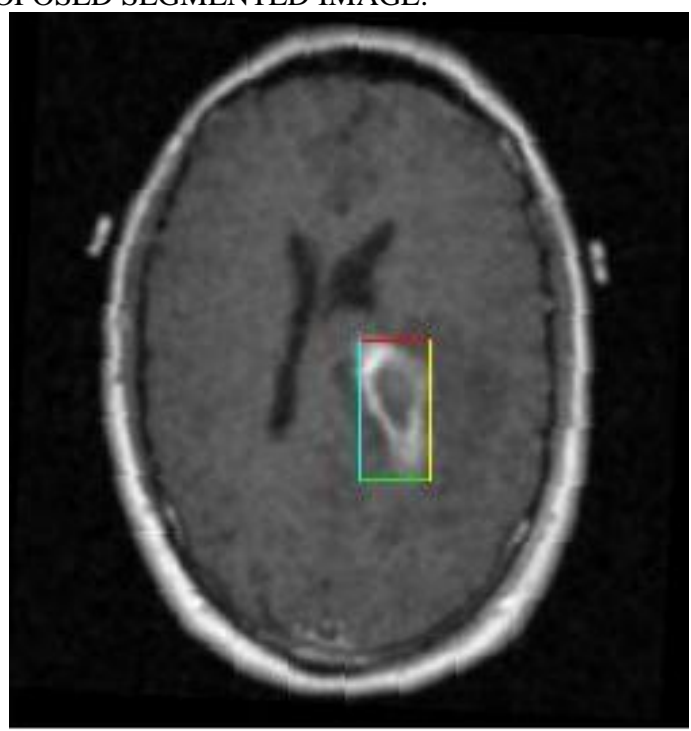

Fig4 :Proposed segmented image 


\section{PERFORMANCE EVALUATION}

Comparison among the proposed and the available algorithms based upon the following parameters:-
a. F-measure
b. Bit error rate
c. Peak signal to noise ratio

True Positive $(\mathrm{TP})=\frac{\text { No. of resulted images having brain tumor }}{\text { Total number of images }}$

True Negative $(\mathrm{TN})=\frac{\text { No. of images that have not tumor }}{\text { Total number of images }}$

False Positive $(\mathrm{FP})=$

No.of images that have not tumor and detected positive

$$
\text { Total no.of images }
$$

False Negative $(\mathrm{FN})=\frac{\text { No.of images have tumor and not detected }}{\text { Total number } \text { of images }}$

Precision $\left(T_{p}\right)=\frac{T P}{T P+F P}$

$\operatorname{Recall}\left(T_{r}\right)=\frac{T P}{T P+F N}$

\subsection{F-Measure}

F-Measure $\left(F_{m}\right)=\frac{100 \times 2\left(T_{p} \times T_{r}\right)}{\left(T_{p}+T_{r}\right)}$

Table 1: Comparison of F-Measure

\begin{tabular}{|l|l|l|}
\hline IMAGES & BASE PAPER & PROPOSED \\
\hline 1 & 69.6785 & 99.0988 \\
\hline 2 & 93.0916 & 99.0081 \\
\hline 3 & 74.0448 & 99.2771 \\
\hline 4 & 52.8691 & 99.7685 \\
\hline 5 & 84.7985 & 99.2644 \\
\hline 6 & 97.8274 & 99.6797 \\
\hline 7 & 62.6332 & 99.5197 \\
\hline 8 & 97.9505 & 99.6187 \\
\hline 9 & 59.1858 & 99.4759 \\
\hline 10 & 61.3379 & 99.6044 \\
\hline 11 & 58.7748 & 99.6138 \\
\hline
\end{tabular}

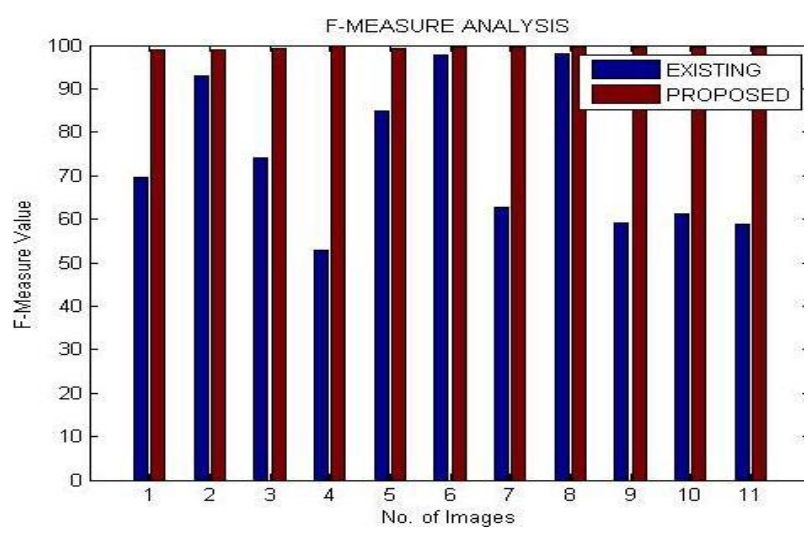

Graph 1:F-Measure Analysis

\subsection{Bit Error Rate}

Bit Error Rate $(\mathrm{BER})=\frac{1}{P S N R}$

Table 2: Comparison of BER

\begin{tabular}{|c|c|c|}
\hline IMAGES & BASE PAPER & PROPOSED \\
\hline 1 & 0.0306 & 0.0283 \\
\hline 2 & 0.0271 & 0.0224 \\
\hline 3 & 0.0303 & 0.0274 \\
\hline 4 & 0.0324 & 0.0217 \\
\hline 5 & 0.0282 & 0.0274 \\
\hline 6 & 0.0248 & 0.0224 \\
\hline 7 & 0.0314 & 0.0245 \\
\hline 8 & 0.0244 & 0.0236 \\
\hline 9 & 0.0318 & 0.0253 \\
\hline 10 & 0.0314 & 0.0237 \\
\hline 11 & 0.0319 & 0.0238 \\
\hline
\end{tabular}

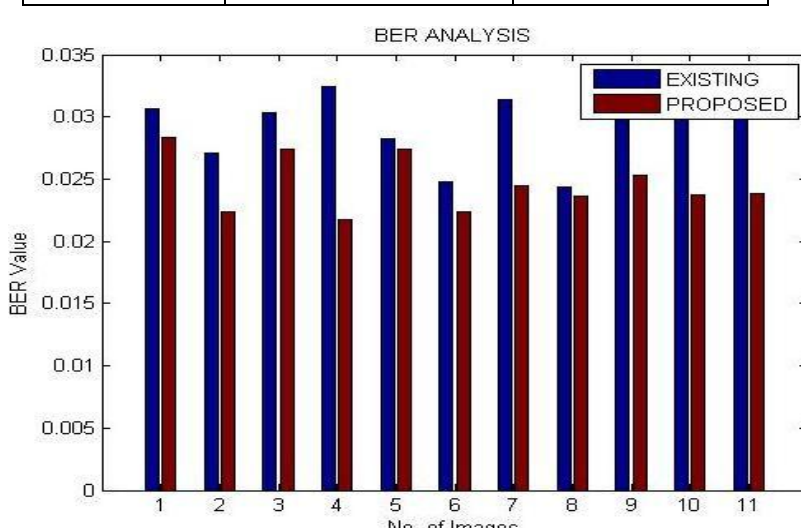

Graph 2: BER Analysis 


\subsection{Peak signal to noise ratio (PSNR)}

Peak Signal To Noise Ratio $(\mathrm{PSNR})=10 \log \frac{255^{2}}{M S E}$

Where MSE denotes mean square error

Table 3: Comparison of PSNR

\begin{tabular}{|l|l|l|}
\hline IMAGES & BASE PAPER & PROPOSED \\
\hline 1 & 32.7253 & 35.3919 \\
\hline 2 & 36.8888 & 34.3775 \\
\hline 3 & 33.0291 & 37.0727 \\
\hline 4 & 30.9022 & 46.8453 \\
\hline 5 & 35.4357 & 36.9134 \\
\hline 6 & 40.2779 & 44.1591 \\
\hline 7 & 31.8642 & 40.7233 \\
\hline 8 & 41.0123 & 42.5612 \\
\hline 9 & 31.4157 & 39.8211 \\
\hline 10 & 31.8011 & 42.4727 \\
\hline 11 & 31.3884 & 42.4727 \\
\hline
\end{tabular}

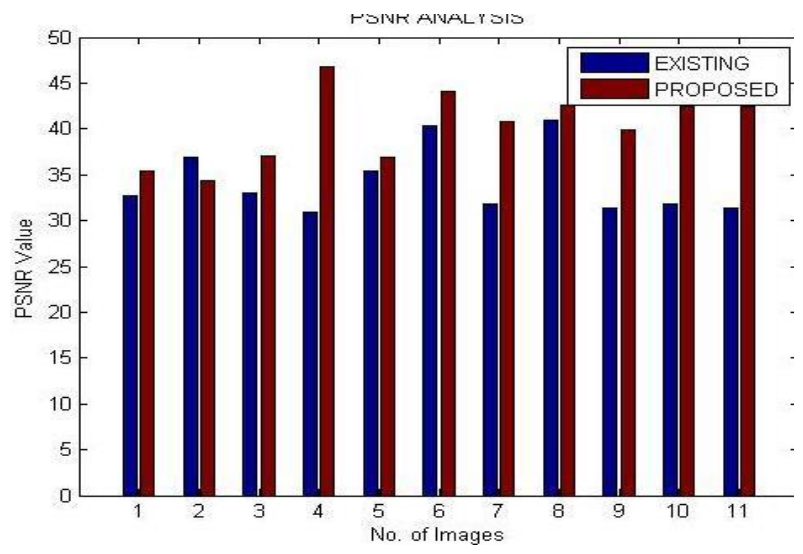

Graph 3: PSNR Analysis

\section{CONCLUSION AND FUTURE SCOPE}

Brain tumor is an abnormal development of cells in the brain of individual body. Typically, the tumor can develop from the cells of the brain, blood vessels, or nerves that appear from the brain. To boost the tumor detection rate further an hybridization of fuzzy and region growing segmentation centered tumor detection with the trilateral filtration is proposed. The proposed approach has the ability to produce effective results even in case of large thickness of the noise. The planned approach was created and implemented in the MATLAB tool along with assistance from image control toolbox. The experimental results has clearly shown that the proposed technique outperforms over the available techniques. This work has not considered the use of particle swarm optimization to segment an image in more proficient manner. So in near future the proposed technique will be enhanced by considering the particle swarm optimization based segmentation.

\section{REFERENCES}

[1] Abdel-Maksoud,Eman,Mohammed Elmogy and Rashid Al-Awadi. "Brain tumor segmentation based on a hybrid clustering technique." Egyptian Informatics Journal (2015).

[2] Perumal, K., and V. G. Karthikram. "A Fusion of SOM and Fuzzy C-Means for Image Processing." International Journal of Computer Technology and Applications 4.4 (2013): 647.

[3] Jose, Alan, S. Ravi, and M. Sambath. "Brain Tumor Segmentation Using K-Means Clustering And Fuzzy CMeans Algorithms And Its Area Calculation."Brain 2.3 (2014).

[4] Dou, W., Ruan, S., Chen, Y., Bloyet, D., and Constans, J. M. (2007), "A framework of fuzzy information fusion for segmentation of brain tumor tissues on MR images", Image and Vision Computing, 25:164-171.

[5] Roy, Sudipta, and Samir K. Bandyopadhyay. "Detection and Quantification of Brain Tumor from MRI of Brain and it's Symmetric Analysis." International Journal of Information and Communication Technology Research 2.6 (2012).

[6] Acharya J, Gadhiya S, Raviya. Segmentation techniques for image analysis: a review. Int J Comput Sci Manage Res 2013;2(4):1218-21.

[7] Naik D, Shah P. A review on image segmentation clustering algorithms. Int J Comput Sci Inform Technol 2014;5(3):3289-93.

[8] Christe SA, Malathy K, Kandaswamy A. Improved hybrid segmentation of brain MRI tissue and tumor using statistical features. ICTACT J Image Video Process 2010;1(1):34-49.

[9] Seerha GK, Kaur R. Review on recent image segmentation techniques. Int J Comput Sci Eng (IJCSE) 2013;5(2):109-12.

[10] Abdel-Maksoud,Eman,Mohammed Elmogy and Rashid Al-Awadi. "Brain tumor segmentation based on a hybrid clustering technique." Egyptian Informatics Journal (2015).

[11] Havaei, Mohammad, P-M. Jodoin, and Hugo Larochelle. "Efficient interactive brain tumor segmentation as within-brain kNN classification." Pattern Recognition (ICPR), 2014 22nd International Conference on. IEEE, 2014.

[12] Preetha, R., and G. R. Suresh. "Performance Analysis of Fuzzy C Means Algorithm in Automated Detection of Brain Tumor." Computing and Communication Technologies (WCCCT), 2014 World Congress on. IEEE, 2014

[13] Dhanalakshmi, P., and T. Kanimozhi. "Automatic segmentation of brain tumor using K-Means clustering and its area calculation." International Journal of advanced electrical and Electronics Engineering 2.2 (2013): 130-134

[14] Kumar, E. Praveen, V. Manoj Kumar, and M. G. Sumithra. "Tumour detection in brain MRI using improved segmentation algorithm." Computing, Communications and Networking Technologies 
(ICCCNT), 2013 Fourth International Conference on. IEEE, 2013.

[15] N. Albert Singh, "Detection of brain tumor using neural network", ICCCNT, 2013, 2013 Fourth International Conference on Computing, Communications and Networking Technologies (ICCCNT), 2013 Fourth International Conference on Computing, Communications and Networking Technologies (ICCCNT) 2013, pp. 1-5, doi:10.1109/ICCCNT.2013.6726524

[16] S. Parisot, "Graph-based detection, segmentation \& characterization of brain tumors", CVPR, 2012, 2013 IEEE Conference on Computer Vision and Pattern Recognition, 2013 IEEE Conference on Computer Vision and Pattern Recognition 2012, pp. 988-995, doi:10.1109/CVPR.2012.6247775

[17] Bhattacharjee, Rupsa, and Monisha Chakraborty. "Brain tumor detection from MR images: Image processing, slicing and PCA based reconstruction."Emerging Applications of Information Technology (EAIT), 2012 Third International Conference on. IEEE, 2012.

[18] Shasidhar, M., V. Sudheer Raja, and B. Vijay Kumar. "MRI brain image segmentation using modified fuzzy cmeans clustering algorithm."Communication Systems and Network Technologies (CSNT), 2011 International Conference on. IEEE, 2011.

[19] Bhat, Subramanya, and Sanjeev R. Kunte. "A mixed model based on Watershed and Active contour algorithms for brain tumor segmentation."Advances in Recent Technologies in Communication and Computing (ARTCom), 2010 International Conference on. IEEE, 2010.

[20] Kannan, S. R., and R. Pandiyarajan. "Effective fuzzy cmean clustering technique for segmentation of T1-T2 brain MRI." Advances in Recent Technologies in Communication and Computing, 2009. ARTCom'09. International Conference on. IEEE, 2009.
[21] Singh, Laxman, R. B. Dubey, and Z. A. Jaffery. "Segmentation and characterization of brain tumor from MR images." Advances in Recent Technologies in Communication and Computing, 2009. ARTCom'09. International Conference on. IEEE, 2009.

[22] Wu, Ming-Ni, Chia-Chen Lin, and Chin-Chen Chang. "Brain tumor detection using color-based k-means clustering segmentation." Intelligent Information Hiding and Multimedia Signal Processing, 2007. IIHMSP 2007. Third International Conference on. Vol. 2. IEEE, 2007.

[23] Mohan P, AL V, Devi BRS, Kavitha BC. Intelligent based brain tumor detection using ACO. Int J Innovat Res Comput Commun Eng 2013;1(9):2143-50.

[24] Anandgaonkar G, Sable G. Brain tumor detection and identification from $\mathrm{T} 1$ post contrast MR images using cluster based segmentation. Int J Sci Res 2014;3(4):814

[25] Jude hemanth.D, D.Selvathi and J.Anitha,"Effective Fuzzy Clustering Algorithm for Abnormal MR Brain Image Segmentation",Page Number609-614, International/Advance Computing Conference (IACC2009),IEEE,2009

[26] Sorin Istrail, "An Overview of Clustering Methods", With Applications to Bioinformatics.

[27] Steven Eschrich, Jingwei Ke, Lawrence O. Hall and Dmitry B. Goldgof Fast "Accurate Fuzzy Clustering through Data Reduction", Page Number 1-18,November 13 IEEE 2002.

[28] Vasuda, P., and S. Satheesh. "Improved fuzzy C-means algorithm for MR brain image segmentation." International Journal on Computer Science and Engineering 2.5 (1713): 2010.

[29] Issac N. Bankman, "Handbook of medical image processing and analysis", Second edition, Academic press, USA, 2008. 